\title{
La temática de las ponencias del III Congreso Latina de Comunicación Social
}

\author{
The thematic of papers presented at the Latina III Congress on Social \\ Communication
}

\author{
Dr. Raymond Colle \\ Ex-profesor Pontificio Universidad \\ Católica de Chile y Universidad \\ Diego Portales Santiago de Chile \\ http://www.recinet.org/colle/rc/
}

Recibido: 21 de diciembre 2011 Aceptado: 25 de enero 2012

\begin{abstract}
Resumen
A partir de los títulos y resúmenes de las ponencias enviadas para el III Congreso Latina y aceptadas al 15 de noviembre 2011, realizamos un análisis semántico basado en la página web que contiene dicha información a fin de extraer la temática cubierta. La metodología se basa en estadísticas: de palabras (originales), de los campos conceptuales en que se han de reagrupar para aislar los temas tratados y de las coocurrencias de dichos campos en la descripción de cada ponencia. Se deduce de ello que la atención se ha dirigido principalmente hacia la política, en términos de contenidos de la comunicación, hacia los medios digitales y especialmente Internet en materia de técnica, y privilegiando el análisis como forma de trabajo. Pero el tema del mercado, sugerido en la convocatoria ha sido mínimamente abordado.
\end{abstract}

\section{Abstract}

Retrieving the titles and abstracts of the papers accepted before the Latina III Congress, we have analyzed the covered thematic. The methodology is based on statistics of words (originals), of the conceptual fields in which they were regrouped to identify the topics discussed and the coocurrences of these fields in the description of each article. So we discover that the attention was most payed on politics, for communication's contents, digital media, specially the internet, for communication's techniques, and with a priotity for analysis as work form. But the issue of the market, suggested in the call has been minimally addressed.

Palabras Clave: Comunicación social, análisis de contenido, red semántica, significado, coocurrencia, concepto, estadística, Anatex, NetDraw, Congreso Latina, 
Key Words: Social communication; semantic analysis; meaning, co-occurrence, concept, Anatex, NetDraw

Sumario: 1. Introducción. 2. Metodología. 2.1. Análisis estadístico. 2.2. Graficación. 2.3 Etapas de análisis. 3. Resultados. 3.1. Términos. 3.2. Temas. 3.3. Coocurrencias. 3.4. La comunicación pública y el mercado. 3.5. El caso de Internet. 4. Conclusiónes. 5. Bibliografía.

Summary: 1. Introduction. 2. Methodology, 3. Statistical analysis. 2.2. Graphing. 2.3. Stages of analysis. 3. Results. 3.1. Terms. 3.2. Themes. 3.3. Coocurrences. 3.4. Public communication and market. 3.5. The Internet case. 4. Conclusions. 5. References.

\section{Introducción}

La Sociedad Latina de Comunicación Social realizó en diciembre 2011 la tercera versión de su congreso internacional. En preparación del mismo, recibió numerosas ponencias y publicó una página web con los títulos y resúmenes de las que fueron aceptadas. El 1 de noviembre eran 175. Aunque se anunció que el plazo para recibir ponencias se extendería por un par de semanas más de lo inicialmente anunciado, habíamos recogido en esa fecha la lista y los resúmenes de estas ponencias, iniciando el análisis de las mismas. Agregamos luego las ponencias publicadas hasta el 15 de noviembre, llegando a $215^{1}$.

La mera lectura no permite descubrir con facilidad cuáles son los temas que han interesado más frecuentemente a los investigadores que se presentan al Congreso. Es lo que nos ha llevado a planear la presente investigación, haciéndonos las siguientes preguntas: ¿Qué temas han sido abordados más frecuentemente? ¿Hay puntos comunes entre los diversos aportes? ¿Se destacan algunas temáticas? ¿Cuáles?

La nuestra es, por lo tanto, una investigación exploratoria, que partió sin ninguna hipótesis definida, como ha de ocurrir muchas veces en el análisis de contenido.

\section{Metodología}

Al plantearnos descubrir la temática cubierta por el Congreso, nos vemos llevados a realizar algún tipo de Análisis de Contenido. No nos extenderemos aquí sobre los aspectos generales de la teoría correspondiente y sus avances históricos desde fines del siglo XIX y más especialmente desde Berelson y Lazarsfeld (1948), con múltiples textos al respecto, como los de Osgood (1957), Holsti (1969), Mucchielli (1974) y Krippendorf (1980) (ver López: 2002 y Marzal y Moreira: 2001). Los desarrollamos, asi como los fundamentos teóricos actuales, en nuestra obra sobre «El análisis de contenido de las comunicaciones» publicada

1 Unas pocas se agregaron después en la web del Congreso y no alcanzaron a entrar en el corpus analizado. 
en los «Cuadernos Artesanos» de «Latina» (2011). Los procedimientos que hemos utilizados son esencialmente estadísticos y gráficos, basados en un análisis automático (computacional).

\subsection{Análisis estadístico}

El análisis estadístico comprende dos partes:

1. Cálculo de frecuencia de aparición (ocurrencia) de palabras. Este tipo de cálculo se utiliza frecuentemente en análisis de contenido, dado que es comunmente aceptado que la frecuencia de las palabras es una medida del grado de importancia de los conceptos aludidos. Así, el mero recuento de los términos utilizados proporciona información significativa en torno a los conceptos priorizados por los autores. Esto, a su vez, es un elemento necesario (pero no necesariamente suficiente) para la definición del diccionario o código conceptual necesario para la etapa final del análisis matemático.

2. Cálculo de coocurrencia de conceptos, como medio para acceder a la descripción del sentido del discurso (o del conjunto textual analizado), extrayendo la estructura semántica subyacente. Para ello, se orienta hacia la búsqueda y el análisis de las interrelaciones de los términos significativos y, de este modo, hacia una explicitación precisa y estructurada de los significados centrales del conjunto.

El análisis de coocurrencia aplicado en el campo del análisis semántico tiene la ventaja de no limitarse a la identificación y suma atomizada de los componentes significativos. Al contrario, le es propio reconocer una importancia fundamental a las relaciones entre dichos componentes, para lo cual considera siempre pares de componentes, midiendo la simultaneidad de su aparición en conjuntos significativos predeterminados. Típicamente, aquí, el conjunto significativo considerado es la oración (definida pragmáticamente como el conjunto de términos que se termina con un punto) y cada par de palabras en el interior de esta unidad gramatical constituye una coocurrencia. Así, si la oración se compone de seis palabras, existirán en ésta quince coocurrencias o pares de palabras. Por cierto el sistema es aplicable a cualquier conjunto con valor semántico, y no sólo a textos. Así, por ejemplo, Hudrisier lo utilizó en Francia para generar sistemas de clasificación de fotografías.

Este procedimiento puede aplicarse a conjuntos de variadas dimensiones (dependiendo de la memoria RAM del ordenador utilizado), obteniendo como resultado una matriz de coocurrencia (tabla de doble entrada en que los conceptos se relacionan unos con otros). Como el tamaño de ésta crece rápidamente, corresponde al analista limitar el número de términos a considerar. Por ello se eliminan todas las palabras no significativas (artículos, pronombres, etc.) y se procede a establecer agrupaciones de términos que se consideraron como formas diferentes de representar un mismo concepto (campos conceptuales). Un campo conceptual se entiende «compuesto por unidades léxicas unidas por relaciones de 
naturaleza diversa en la lengua en uso, ya sea por relación semántica paradigmática, por asociaciones dadas por la cultura o por el conocimiento del mundo». (García: 1990:103). En el presente caso, el software utilizado (Anatex3, ver Colle: 2005) permite definir la cantidad de campos conceptuales que se desee, cada uno a su vez compuesto del número de términos que sea necesario. Esto corresponde a la técnica de los "diccionarios" - como el clásico "Diccionario de Lasswell" y otros - utilizados por múltiples sistemas de análisis computacional del discurso (p.ej. el Minnesota Contextual Content Analysis, cfr. Litkoswski y McTavish:2001).

Después de analizar el superconjunto, se tendrá información acerca todas las coocurrencias reales, lo cual puede poner en evidencia procesos psicológicos subyacentes a la formulación del discurso, estrechamente vinculados al carácter semántico de la comunicación. Estas interrelaciones, además, al unirse entre sí conforman una red, que -al dibujarse- puede poner en evidencia sub-áreas y términos más importantes por su centralidad o por una función de unión entre sub-áreas. Esta técnica se origina en antiguas hipótesis del conductismo sobre el significado de las palabras, mejoradas luego con los aportes del llamado Modelo de Red Semántica de Collins y Quillian, establecido por dichos autores en 1969, pero corregido posteriormente de diversas maneras (cfr. Harley: 2008). El primer programa computacional de representación de una red semántica se debe a Richard H. Richens del Cambridge Language Research Unit (1956), sistema luego desarrollado por Robert F. Simmons en la System Development Corporation en los años 1960. Con la generalización del uso la computación en múltiples disciplinas, en los años ochenta y siguientes, se han multiplicado los trabajos de análisis computacional de redes semánticas. (cfr. Kleinnijenhuis: 2007)

\subsection{Graficación}

El software Anatex que hemos utilizado proporciona las tablas de coocurrencia (tanto la matriz general como el listado de pares asociados) a partir del diccionario conceptual definido por el investigador pero no es capaz de graficar el mapa que representaría las interrelaciones. Para ello se recurrió a NetDraw, un software especializado de gráfica vectorial (Borgatti: 2002). Dos procedimientos son de importancia aquí:

a. la simplificación del grafo, es decir la búsqueda de la óptima ubicación de cada elemento para evitar al máximo el cruce de los arcos (líneas que representan las relaciones de coocurrencia);

b. la selección de «umbrales» donde detener la inclusión o exclusión de nuevos términos en un determinado mapa.

Es evidente que si se incluyen en un mapa todos los conceptos de un discurso, el mapa se hará ilegible y dejaremos de cumplir el objetivo de extraer la estructura organizadora y los subcampos semánticos medulares del mismo. Existe por lo tanto la necesidad de proceder por etapas, partiendo por las coocurrencias más frecuentes y construyendo varios grafos, correspondientes a diferentes niveles de frecuencia o bien, a la inversa, graficar toda la red y

Facultad de Ciencias de la Información - Universidad de La Laguna

Avenida César Manrique, s/n; Campus de Guajara

38071 La Laguna, Tenerife (Islas Canarias - España) 
esconder los vínculos menos frecuentes. El software de análisis de redes, como NetDraw, procede generalmente de este modo, registrando la totalidad de la red, y ofrece procedimientos específicos para el análisis de ésta, como la selección sobre la base de atributos (como ciertas características de los integrantes o categorías que se hayan establecido previamente) y, en particular, del peso relativo de los vínculos (frecuencias) entre los nodos (integrantes de la red). Así, se pueden seleccionar umbrales de frecuencia que ponen en evidencia grupos, subgrupos y elementos tanto centrales como marginales ( $y$, por lo tanto, poco significativos).

\subsection{Etapas de análisis}

En una primera etapa se seleccionó todo el texto de la página web del Congreso que presentaba las ponencias aprobadas al término del plazo de recepción (15 de noviembre), siendo éstas 215, el cual constituye el corpus principal. Se suprimieron el encabezado y los nombres y datos de autores, de tal manera que quedara solamente información relativa al contenido de las ponencias y se dio a este texto el formato requerido para el análisis automático («corpus»). Paralelamente, se seleccionaron los títulos y subtítulos de las ponencias cuyo conjunto formó un corpus secundario, ajustando también su formato.

Se pasó luego a la segunda etapa, consistente en un análisis automático de frecuencia de las palabras contenidas en el corpus principal (eliminando automáticamente las palabras vacías como artículos, preposiciones, pronombres, etc.). La lista obtenida sirvió básicamente para verificar la adecuación del diccionario conceptual a utilizar en la siguiente fase. Recurrimos al «diccionario» ya definido y utilizado en nuestro anterior análisis de «La temática de la Revista Latina de Comunicación Social 1998-2008», al cual solo tuvimos que agregar un par de conceptos (Ver Anexo 1). Estos conceptos (o más exactamente campos conceptuales) son los «temas» de las ponencias que nos interesa conocer. Esta fase es obviamente la más subjetiva del trabajo, aunque se basa en algunos principios semánticos. (Véase el Anexo 1 para juzgar).

Disponiendo de este «diccionario», se aplicó en forma automática a cada corpus un análisis estadístico de los campos presentes. Los resultados de este análisis fueron vertidos a una hoja de cálculo para poder transferirlos al presente informe.

Posteriormente se pasó al análisis de coocurrencia, consistente en crear una matriz en que se suman las coocurrencias de todos los campos conceptuales, la unidad de análisis siendo el conjunto de palabras correspondiente a cada oración (o frase, en el caso de los títulos y sub-títulos) de cada ponencia. En otras palabras, se registran todas las palabras del conjunto, se verifican cuales están en el «Diccionario» y se suma el caso en el registro del cruce de cada campo conceptual con los demás campos aludidos en la misma unidad analizada (oración o frase). Los resultados de esta etapa están constituídos por pares de campos conceptuales con su respectiva frecuencia de coocurrencia (Ver ejemplos más 
adelante).

Contando con estos pares y su frecuencia, pasamos a representar las relaciones entre campos conceptuales (los «temas») en forma gráfica, tal como se hace en sociometría para representar las relaciones grupales y las estructuras de liderazgo (análisis de redes). Se obtiene así un mapa semántico, que representa no sólo los campos más frecuentes (cosa que se puede lograr con el primer análisis estadístico mencionado, pero de baja significación semántica) sino más particularmente la estructura de interrelaciones y el grado de centralidad de estos campos conceptuales en el discurso total analizado, lo cual no aparece siempre a la vista por mera lectura.

Los cálculos se realizaron utilizando el software ANATEX, una aplicación desarrollada originalmente en la Pontificia Universidad Católica de Chile (en 1988 para la plataforma Macintosh y actualizada por este autor en 2005 para el sistema operativo Linux), destinada a facilitar operaciones vinculadas al análisis de textos, tanto en el campo del análisis de contenido como en lingüística y otras áreas vinculadas a las anteriores como la hermenéutica y la exégesis. Realizados los dos tipos de operaciones de cálculo necesarios en la presente investigación -primero la estadística de palabras y luego la estadística de campos conceptuales concurrentes- pasamos a la graficación utilizando la aplicación NetDraw (Borgatti, 2002).

\section{Resultados}

\subsection{Términos}

Sumados todos los títulos y resúmenes -pero excluida la identificación de los autores- hemos registrado más de 53.000 palabras, de las cuales 3.640 en títulos y sub-títulos ${ }^{2}$. Para el análisis, se eliminaron en forma automática las llamadas «palabras vacías» (artículos, preposiciones, cifras, etc.) quedando solamente las palabras significativas.

Confeccionar una estadística total de las palabras, debido a la cantidad registrada, era imposible para nuestro procesador. Calculamos solamente las frecuencias de las palabras originales en los títulos y subtítulos, lo cual nos arrojó los resultados señalados en la Tabla 1, donde recogemos solamente las frecuencias mayores de 5.

2 El número de cada ponencia ha sido contado como una palabra.

Facultad de Ciencias de la Información - Universidad de La Laguna

Avenida César Manrique, s/n; Campus de Guajara

38071 La Laguna, Tenerife (Islas Canarias - España) 


\section{Tabla 1: Términos más frecuentes en títulos}

\begin{tabular}{|c|c|}
\hline Términos & Frec. \\
\hline comunicación & 44 \\
\hline análisis & 33 \\
\hline prensa & 20 \\
\hline periodismo & 17 \\
\hline información & 16 \\
\hline medios & 15 \\
\hline política & 13 \\
\hline publicidad & 13 \\
\hline caso & 13 \\
\hline televisión & 12 \\
\hline España & 11 \\
\hline cine & 11 \\
\hline nuevos & 11 \\
\hline española & 10 \\
\hline estudio & 10 \\
\hline audiovisual & 10 \\
\hline social & 10 \\
\hline discurso & 10 \\
\hline investigación & 9 \\
\hline producción & 9 \\
\hline cultura & 8 \\
\hline digital & 8 \\
\hline ficción & 7 \\
\hline informativos & 7 \\
\hline metodología & 7 \\
\hline nuevas & 7 \\
\hline relación & 7 \\
\hline web & 6 \\
\hline
\end{tabular}

Fuente: Elaboración Propia

A partir de estas palabras, reagrupándolas, hemos ajustado la lista de temas (campos conceptuales) abordados. Ya hemos señalado que hemos utilizado la lista («diccionario») generada en nuestro análisis de la temática de la Revista Latina (2009). A la vista del texto de los resúmenes de las ponencias, hemos agregado a dicho diccionario un par de temas que no existían en éste y sí parecían frecuentes en el nuevo corpus como « 2.0 y «red social».

Es indispensable recordar que los temas consisten, en múltiples casos, en la reagrupación de múltiples términos (por lo que, formalmente, se habla más bien de "campos 
conceptuales»). Así, por ejemplo, el campo o tema «educación» reagrupa doce términos asociados:

$$
\begin{aligned}
& \text { educación = \{ educación, educar, educativa, educativo, docencia, enseñanza, escolar, } \\
& \text { escolares, estudiantes, formación, profesor, profesorado \} }
\end{aligned}
$$

Al contrario, ante la -esperable- alta cobertura del tema del periodismo, preferimos subdividirlo en dos subtemas:

- periodismo $=\{$ fotoperiodismo, periodismo, periodística, periodístico, periodista, periodistas $\}$

- prensa $=\{$ diario, diarios, imprenta, impreso, impresos, periódico, periódicos, prensa, publicaciones, revista, revistas, semanario \}

\subsection{Campos conceptuales (Temas)}

Analizados primero los títulos (y eventuales subtítulos) de las ponencias, para obtener una primera visión de conjunto de las temáticas abordadas, utilizando el «diccionario» predefinido para extraer los campos conceptuales (que fueron 62). Las frecuencias superiores a 1 se presentan en la Tabla 2, columna 2.

\begin{tabular}{lcc}
\multicolumn{3}{l}{ Tabla 2: Temas más frecuentes en títulos } \\
\cline { 2 - 3 } $\begin{array}{l}\text { Temas } \\
\text { comunicación }\end{array}$ & Frec. en títulos & Frec.total \\
\cline { 2 - 3 } análisis & 33 & 348 \\
información & 31 & 144 \\
prensa & 31 & 168 \\
España & 27 & 150 \\
nuevo & 26 & 115 \\
periodismo & 23 & 161 \\
política & 21 & 122 \\
medio & 18 & 141 \\
publicidad & 17 & 119 \\
educación & 16 & 104 \\
cine & 14 & 67 \\
televisión & 14 & 106 \\
digital & 13 & 60 \\
estudio & 13 & 141 \\
social & 13 & 116 \\
audiovisual & 10 & 55 \\
elección & 9 & 43 \\
internet & 9 & 99 \\
cultura & 8 & 87 \\
investigación & 8 & 118 \\
metodología & 7 & 54
\end{tabular}




$\begin{array}{llc}\text { público } & 7 & 100 \\ \text { discurso } & 6 & 52 \\ \text { iconografía } & 6 & 51 \\ \text { local } & 6 & 34 \\ \text { México } & 6 & 21 \\ \text { red-social } & 6 & 46 \\ \text { web } & 6 & 46 \\ 2-0 & 5 & 24 \\ \text { democracia } & 5 & 14 \\ \text { Canarias } & 4 & 9 \\ \text { desarrollo } & 4 & 51 \\ \text { narrativa } & 4 & 21 \\ \text { radio } & 4 & 22 \\ \text { tecnología } & 4 & 29 \\ \text { economía } & 3 & 24 \\ \text { ética } & 3 & 34 \\ \text { guerra } & 3 & 6 \\ \text { historia } & 3 & 24 \\ \text { identidad } & 3 & 23 \\ \text { industria } & 3 & 15 \\ \text { local } & 3 & 34 \\ \text { noticia } & 3 & 52 \\ \text { teoría } & 3 & 26 \\ \text { ciencia } & 2 & 38 \\ \text { El-País } & 2 & 12 \\ \text { mensaje } & 2 & 41 \\ \text { mercado } & 2 & 41\end{array}$

Fuente: Elaboración propia

Luego hicimos la misma operación con el corpus completo, es decir de los títulos, subtítulos y resúmenes, ya que en éstos se explicitarían más los temas abordados y aparecerían más relaciones entre ellos y con otros no citados en los títulos. Aquí, se obtuvo una frecuencia total de temas aludidos de 3.639 y promedio por tema de 58,7 veces, aunque solo son 20 los temas que superan este promedio. Las frecuencias obtenidas se agregaron en la tabla 2 , columna 3.

Queda claro que se produce una variación, dado el mayor nivel de detalle. Sin embargo, 8 de los 10 temas más frecuentes coinciden. En la Tabla 3, mostramos las frecuencias obtenidas para el corpus total, en orden descendente, con el porcentaje respectivo, limitando la lista a las frecuencias superiores al promedio $(58,7)$. El Gráfico 1, que abarca el total, permite observar la rápida disminución de las mismas.

Tabla 3. Temas más frecuentes en el corpus $(F>58,7)$ 


$\begin{array}{lcc}\text { Tema } & \text { Frec. } & \text { \% }^{*} \\ \text { comunicación } & 348 & 9,56 \\ \text { medio } & 219 & 6,02 \\ \text { información } & 168 & 4,62 \\ \text { nuevo } & 153 & 4,42 \\ \text { prensa } & 150 & 4,12 \\ \text { análisis } & 144 & 3,96 \\ \text { política } & 141 & 3,87 \\ \text { estudio } & 141 & 3,87 \\ \text { periodismo } & 122 & 3,35 \\ \text { investigación } & 118 & 3,24 \\ \text { social } & 116 & 3,19 \\ \text { España } & 115 & 3,16 \\ \text { publicidad } & 113 & 3,11 \\ \text { televisión } & 106 & 2,91 \\ \text { educación } & 104 & 2,86 \\ \text { público } & 100 & 2,75 \\ \text { internet } & 99 & 2,72 \\ \text { cultura } & 87 & 2,39 \\ \text { cine } & 67 & 1,84 \\ \text { digital } & 60 & 1,65\end{array}$

* Estos porcentajes se han establecido en base al total de temas citados, incluyendo las repeticiones de los mismos en una misma ponencia.

Fuente: Elaboración propia

\section{Gráfico 1: Temas por frecuencia}

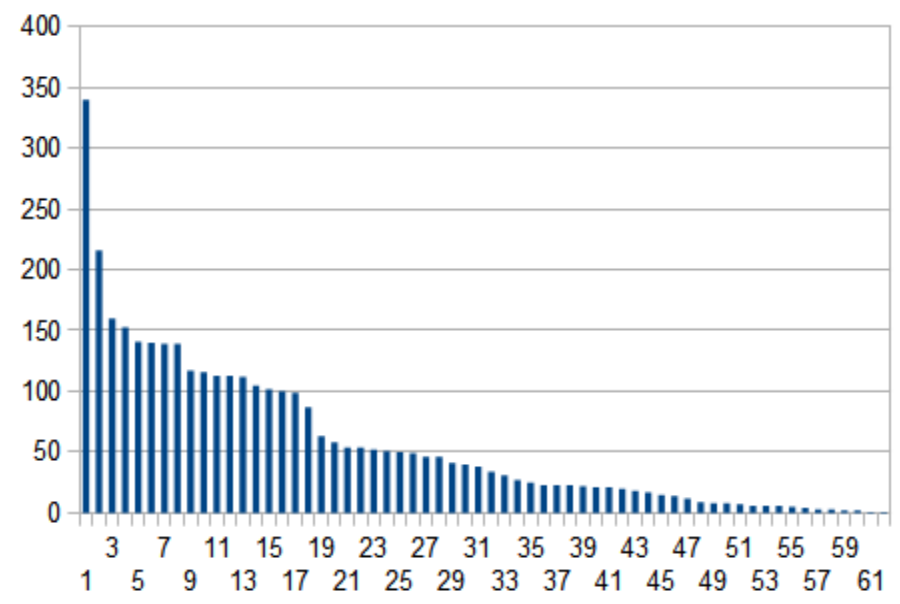

Fuente: Elaboración propia

Como era de esperar, los conceptos más frecuentes son los directamente vinculados a la esencia del Congreso: la comunicación, los medios y la información. Luego aparece una 
preocupación fuerte por lo que está ocurriendo en este ámbito, señalándose lo «nuevo» como primer tema más específico. Aunque represente solo el 4,42\% de todas las menciones, una frecuencia neta de 161 indica su presencia en una gran cantidad de ponencias (recordemos que analizamos 215). [Nos referimos aquí a la totalidad del corpus.]

Llama la atención que el tema político aparece como el primer contenido específico tratado, aunque solo sea con un 3,87\% pero una frecuencia alta de 141 . La publicidad es el contenido más mencionado $(3,11 \%)$ después de la política.

En materia de medios de comunicación señalados, aparece primero la prensa $(4,12 \%)$ a lo cual podríamos quizás agregar el 3,35\% del periodismo, lo cual transformaría «lo periodístico» en el tema más importante, con 7,47\%. Después de la prensa, es la televisión que aparece más mencionada como medio específico (2,91\%). «Internet» se menciona en el $2,72 \%$, pero -para un análisis más fino de esta nueva realidad- hemos considerado aparte «web», «red social» y «2.0», que aportan 1,26\%, 1,26\% y 0,63\% respectivamente, lo cual arroja un total de 6,37\%, lo cual acerca esta temática a la de «lo periodístico» y la transforma en una de las dos más relevantes, más aún si se le agrega «digital» (1,66\%), lo cual daría a la «galaxia digital» un destacado y dominante $8,03 \%$. El cine y diversas formas audiovisuales también han sido mencionados con cierta frecuencia.

Enfocándonos en el tipo de trabajo al cual aluden las ponencias, se destaca que el «análisis» aparece como la mayor alusión a un método, con 3,96\%, a lo cual hay que agregar 0,49\% para «análisis de contenido», que hemos registrado en forma separada, por razón de interés personal. Así las menciones de un trabajo de análisis alcanzan el $4,45 \%$ de los temas mencionados. Menos específico es «Investigación», que aparece en el 3,24\% de los casos. También ha de considerarse una parte del porcentaje que corresponde a «estudio» (3,87\% en total). Como el cálculo automático no permite la desambiguación de este término, tuvimos que realizarla manualmente, encontrando que se utilizó 1 vez en el sentido de «estudios de filmación» y 5 veces en la acepción ligada a la enseñanza. Así, apareció 112 veces con un significado cercano a «investigación», o sea 3,16\%.

Como localización de los estudios, cuando corresponde, se destaca España (115 menciones y 3,24\%), lo cual es bastante lógico dado el lugar del Congreso. Esto es evidentemente mucho más patente al considerar solamente los títulos y lo sería aún más si le sumáramos las alusiones a diversas comunidades autónomas o ciudades españolas.

El tema de la enseñanza-aprendizaje («educación») aparece en el 2,86\% del total, en $15^{\circ}$ posición (porcentaje que sube a $3,38 \%$ si se le agregan los 5 casos en que «estudio» tuvo este significado). Sin duda es relevante en una reunión de académicos. 


\subsection{Coocurrencia de temas}

Dado que la descripción de las ponencias implicaba el uso de múltiples términos, era importante analizar la interrelación de los temas señalados en cada caso. Es lo que se obtiene mediante el análisis de la coocurrencia de éstos (análisis semántico de red), el que arrojó los resultados que presentamos en la Tabla 4, comparando nuevamente lo ocurrido al considerar solamente los títulos y subtítulos versus el corpus completo. El orden dado aquí es el correspondiente a las frecuencias totales iguales o superiores a 10.

En el total del corpus, encontramos 21 pares concurrentes que aparecen más de 20 veces y 31 de 10 a 20 veces, mientras cerca de 400 pares concurrentes aparecen una sola vez.

El primer caso muestra una asociación bastante obvia, entre «comunicación» y «medio». Sin embargo, no significa necesariamente «medio de comunicación»: la asociación significa solamente que los dos temas aparecen en una misma frase, sea unidos con la preposición «de» sea de otra forma. Lo mismo vale para «comunicación social» y casos semejantes. Es de esta forma que deben interpretarse todos los resultados relativos a la coocurrencia.

La principal observación es que la repetición de los mismos pares asociados es bajísima, el par -que es el más frecuente- ocupando solo el 3,08\% del total de pares registrados, lo cual da cuenta de la diversidad de la redacción (más que de las ponencias, ya que la unidad de análisis ha sido la oración y el corpus considerado aquí el conjunto de títulos y resúmenes). En relación a las ponencias se puede hacer una estimación de diversidad dividiendo la frecuencia neta total por la cantidad de ponencias, lo cual arroja un promedio de $40,47 \%$ para «comunicación-medio», y 17,2\% para «comunicación-política» pero estos valores son solo aproximaciones ya que algunas ponencias podrían haber utilizado dichos pares varias veces.

La principal utilidad de este tipo de cálculo es la posibilidad de confeccionar una suerte de mapa semántico de las relaciones (técnica de análisis de red) entre los conceptos, de acuerdo a la formulación realizada por los autores. Las relaciones se observan mejor cuando se construye, gráficamente, la red de interrelaciones, siempre que se limiten los arcos (líneas que muestran las relaciones) en función de la frecuencia.

Tabla 4. Coocurrencias más frecuentes $(>10)$

$\begin{array}{cc}\text { Prec. en coocurrente } & \text { Frec.total } \\ \text { títulos } & \end{array}$

$\begin{array}{llll}\text { comunicación } & \text { medio } & 9 & 87 \\ \text { comunicación } & \text { información } & 2 & 40 \\ \text { comunicación } & \text { nuevo } & 3 & 39 \\ \text { comunicación } & \text { política } & 5 & 37 \\ \text { análisis } & \text { estudio } & 4 & 36\end{array}$

Facultad de Ciencias de la Información - Universidad de La Laguna Avenida César Manrique, s/n; Campus de Guajara 38071 La Laguna, Tenerife (Islas Canarias - España) 


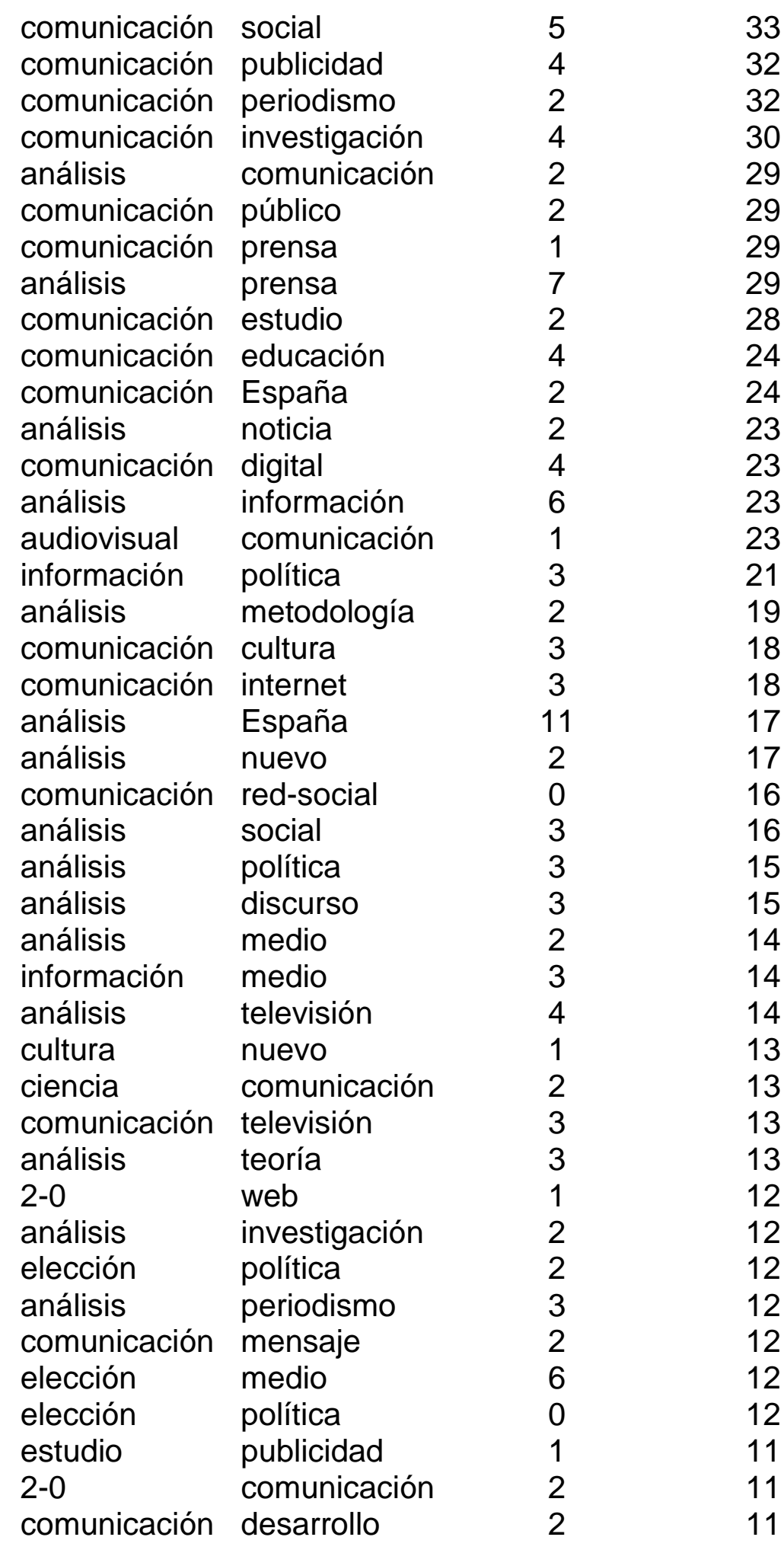


Fuente: Elaboración propia

Así, hicimos un primer gráfico a partir de las coocurrencias presentes en el conjunto de títulos y subtítulos. El análisis con NetDraw nos llevó al gráfico 2. Se han descartado los vínculos que aparecen una sola vez. Se observa claramente la importancia central de los concetos de comunicación, análisis e información, así como la referencia a España, ya señalada al comentar la frecuencia individual de los temas (4.1).

\section{Gráfico 2: Interrelaciones temáticas en títulos}

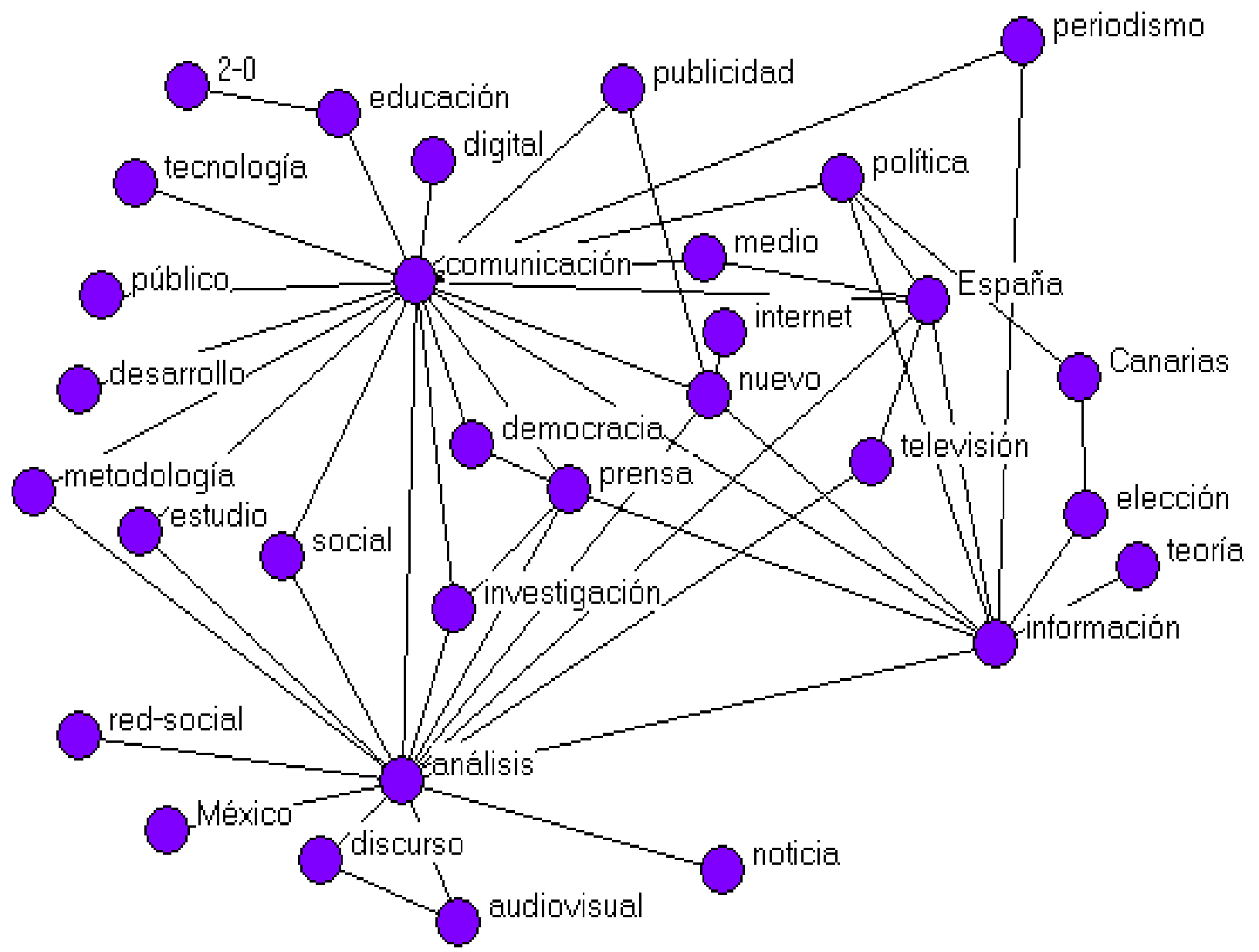

Fuente: Elaboración propia

El siguiente gráfico (3), también construido con NetDraw, se basa esta vez en la totalidad del corpus y muestra todos los casos con frecuencia superior a 10, en concordancia con la Tabla 
2. Se observa nuevamente el rol central y aglutinador de los temas «comunicación» y «análisis», también presentes en el gráfico anterior. Pero algunas asociaciones son menos potentes (como en el caso de «información» y «prensa», por ejemplo) mientras otras ganan en importancia. Es evidentemente difícil comparar los dos gráficos, especialmente porque la ubicación de los vértices varía. Las principales diferencias son más fáciles de apreciar en la tabla de frecuencias. Más que un propósito de comparación, la presentación de los dos gráficos pretende más bien ofrecer al lector la posibilidad de elegir el que le acomode mejor para tener una visión de lo tratado en el congreso, sea a través de la enunciación breve (títulos) sea a través de una descripción más extensa.

\section{Gráfico 3: Interrelaciones temáticas (>10)}

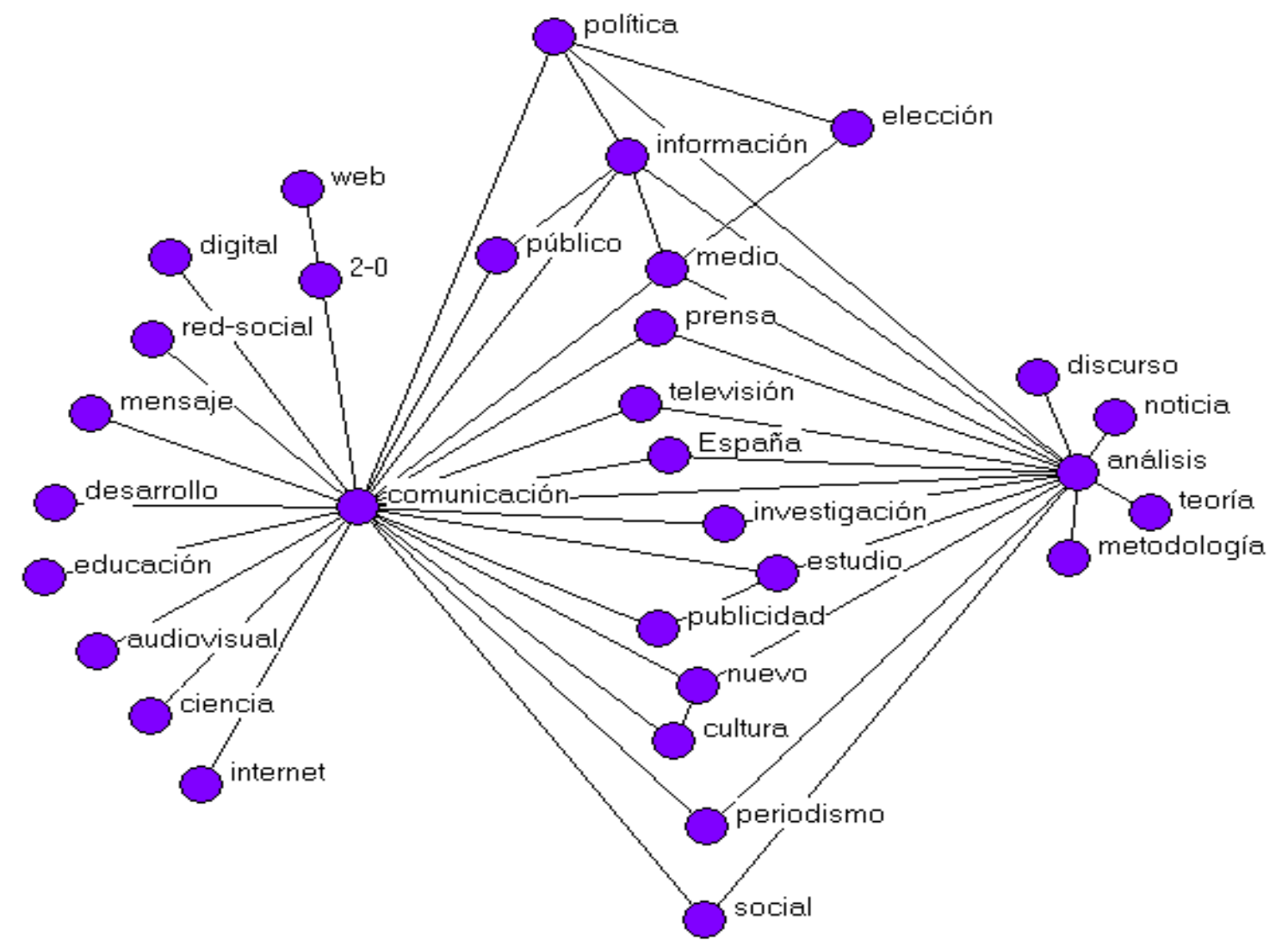

Fuente: Elaboración propia 


\section{Gráfico 4: Interrelaciones temáticas mayores (>17)}

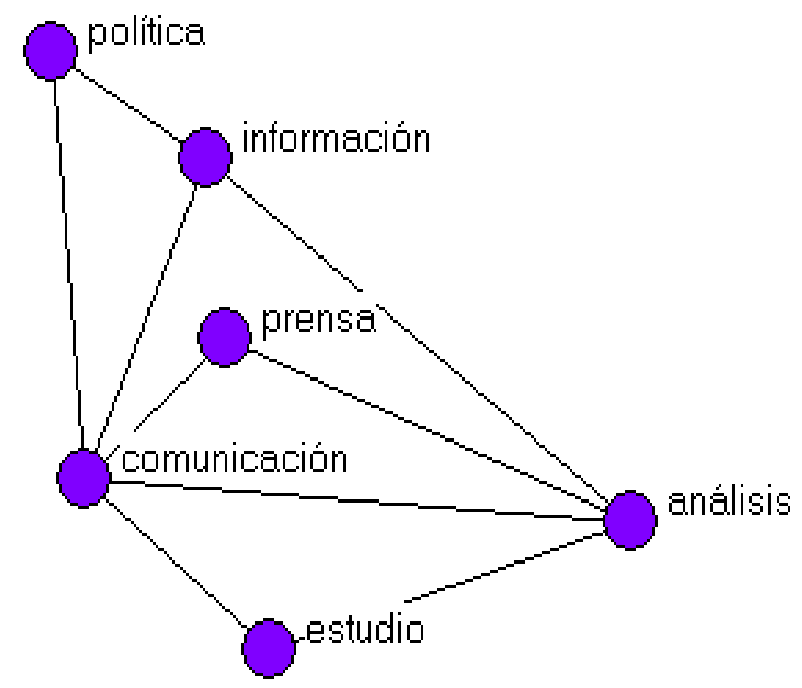

Fuente: Elaboración propia

Seguimos el análisis gráfico considerando solamente los datos más completos (total del corpus). Así, el gráfico 4 reduce los anteriores a los temas de más alta frecuencia que mantienen fuertes relaciones entre sí (frecuencia de coocurrencias superior a 17), quedando aún más en evidencia la importancia dada a la política como contenido, así como a la consideración de la prensa, cosas que no se deducen fácilmente de los gráficos anteriores.

En todos los casos graficados, la elección de los umbrales (diferenciación entre lo mostrado y lo descartado) se basa en criterios técnicos como la estructura del propio gráfico, las frecuencias de correlaciones y la existencia de los arcos (vínculos), a través de las herramientas de NetDraw.

\subsection{La comunicación pública y el «mercado»}

Hicimos este ejercicio de reducción para verificar en qué medida las ponencias se han concentrado en torno al tema central propuesto por el congreso, en este caso «La comunicación pública, secuestrada por el mercado». Si bien el concepto de "comunicación» es central, parece serlo más por el ámbito global o área disciplinaria que por otro motivo y, si bien lo «público» está presente en los mapas de altas frecuencias tanto de títulos como del conjunto, no es todo lo central que se podía esperar ya que no se mantiene en el gráfico 4 . El «mercado» se mencionó solo 2 veces en los títulos, y apareció 41 veces en el corpus total, lo que lo sitió al final de la Tabla 2.

El par «comunicación-público» aparece solo 2 veces en títulos y 29 veces en el corpus total. El par «comunicación-mercado» aparece solo 10 veces en el corpus total y «públicomercado» no aparece ni en los títulos ni en el corpus general, lo cual significa que la tríada 
«comunicación-público-mercado», que corresponde al tema central de la convocatoria, está totalmente ausente del conjunto estudiado.

\subsection{El caso de «Internet»}

La actualidad se hace esperadamente presente en el abordaje del tema de internet, la web, las redes sociales y todo el fenómeno «2.0». Por esta razón, hemos extraído estos cuatro temas y buscado con cuales se relacionan en las diversas ponencias (considerando el corpus total). El gráfico 6 exhibe dichas relaciones. El grosor de los arcos señala aquí los vínculos más frecuentes, que son este caso red-social-comunicación-internet, web-2.0comunicación y análisis-internet. (En este gráfico están presentes todas las frecuencias superior a 1, desde 16 para red-social-comunicación hasta internet-red-social que solo tiene 2, como muchos otros casos).

Si extraemos el «núcleo» -las relaciones más fuertes-, el procedimiento nos muestra que el interés está ligado a la novedad («nuevo») de este tipo de comunicación, lo cual lleva a los autores a un «análisis» o un «estudio» de la situación, mencionado especialmente la «web» «2.0» y las redes sociales (Gráfico 6). Puede llamar la atención el que practicamente no se mencionen juntos la web y las redes sociales (Gráficos 5 y 6). 


\section{Gráfico 5: Temas asociados a Internet}

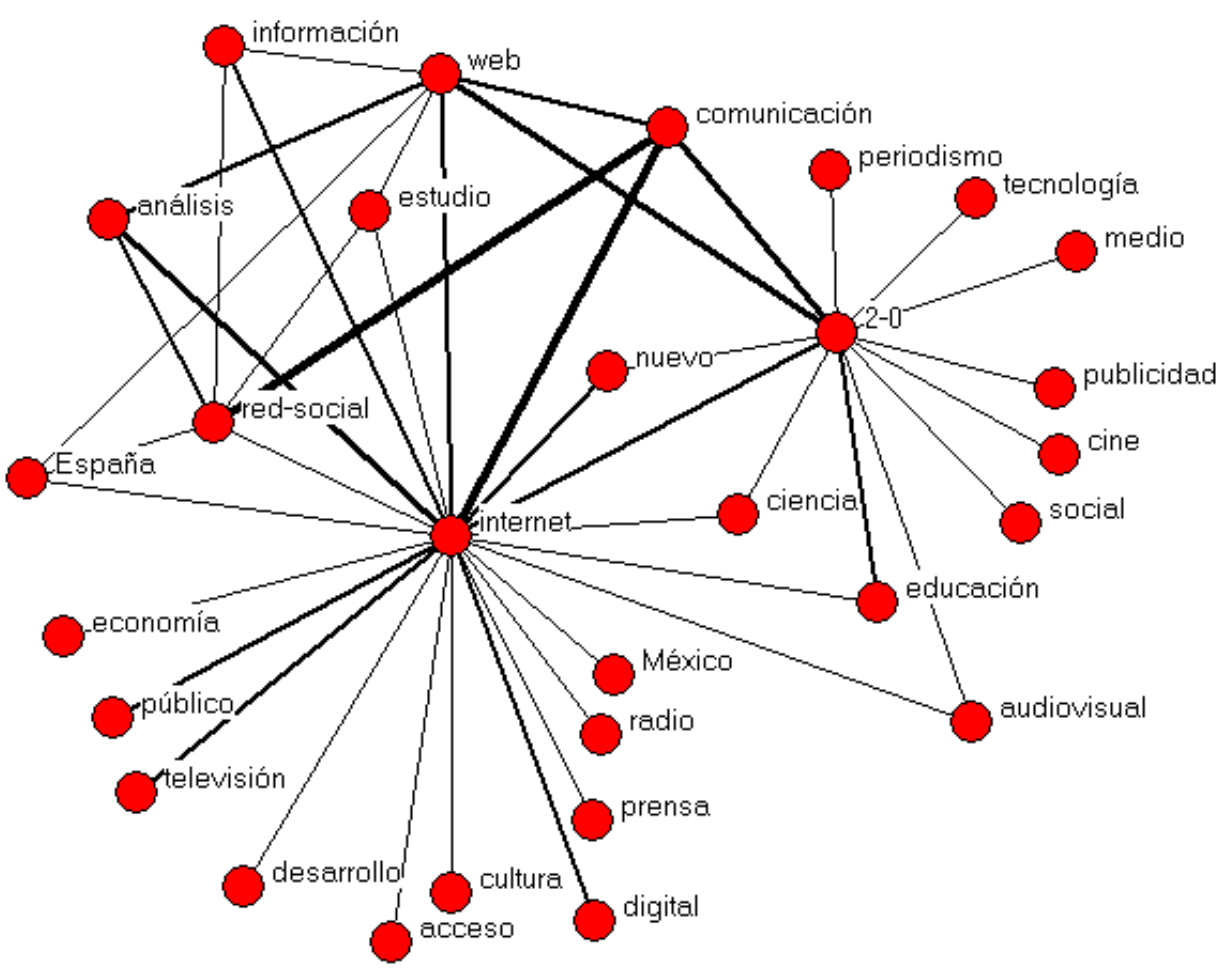

Fuente: Elaboración propia

\section{Gráfico 6: Aspectos centrales en el tratamiento de Internet}

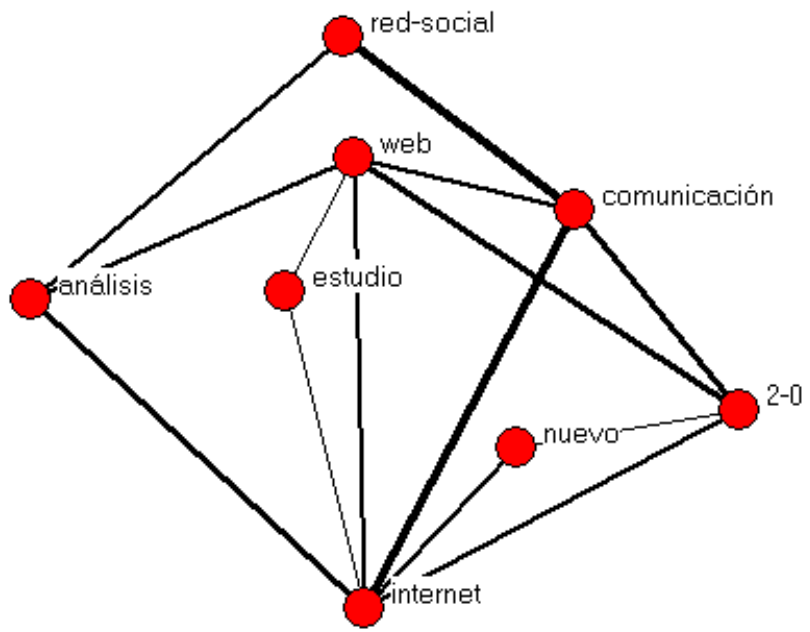

Fuente: Elaboración propia 


\section{Conclusiones}

Como era de esperar, los resultados indican que los temas abordados son altamente coherentes con los objetivos generales del Congreso pero no así con la temática central sugerida para su tercera versión. Si bien era lógico que aparecieran con las más altas frecuencias los relativos a la comunicación, la información y los medios, no se podía tan fácilmente prever la importancia de la política como contenido, por sobre la del mercado mencionada en la convocatoria. Internet era un tema esperado debido a la coyuntura, y se nota que se le está dando gran importancia, especialmente en las funciones sociales de la web.

En síntesis, la atención de los participantes en el III Congreso Latina de Comunicación Social se ha dirigido principalmente hacia la política, la prensa y el periodismo, y las nuevas tecnologías digitales-sociales (Internet/web 2.0). Dado que el congreso se realizó en España, convocado por una revista y una sociedad de profesionales principalmente españoles, no es de extrañar que España sea también el país que ha concitado más atención.

\section{Bibliografía}

Borgatti, S.P., (2002): NetDraw: Graph Visualization Software, Harvard: Analytic Technologies.

Colle, R., (2011): El análisis de contenido de las comunicaciones, Cuadernos Artesanos n¹1, 12 y 13, La Laguna, Tenerife. (http://www.revistalatinacs.org/067/cuadernos/artesanos.html)

Colle, R., (2009): La temática de la Revista Latina de Comunicación Social, en Revista Latina de CS 64, 2009 (http://www.revistalatinacs.org/09/art/07_806_13_revista/Raymond_Colle.html)

Colle, R., (2005): Anatex 3.3, Software de análisis de coocurrencias para plataforma LAMP (Linux-Apache-MySql-PHP), Santiago de Chile: Ver detalles en http://www.recinet.org/colle/anatex.htm

Colle, R., (2002): Explotar la información noticiosa, Madrid: Universidad Complutense, Depto. de Biblioteconomía y Documentación. (agotado; versión PDF disponible con el autor)

Dürsteler, J.C., (2004): «Mapas Conceptuales», en Infovisnet mensaje 141, http://www.infovis.net/Revista/num 141

Gaines, B. \& Shaw, M.: Concept Maps as Hypermedia Components, Calgary: University of Calgary, http://ksi.cpsc.ucalgary.ca/articles/ConceptMaps/ (no fechado)

García Gutiérez., A., (1990): Estructura lingüística de la documentación, Murcia: Ed.Universidad de Murcia. 
Harley, T. A. (2008). Word meaning, East Sussex, UK: Psychology Press.

Kleinnijenhuis, J., (2007): Semantic network analysis: old ideas and new means to master the information society, Universidad de Amsterdam, http://www.ictonderzoek.net/3/assets/File/SIREN\%202008/SIREN2008 Kleinnijenhuis.pdf

Litkowski, K. \& McTavish, D., (2001): Minnesota Contextual Content Analysis system, http://www.clres.com/cata/

López, F., (2002): «El análisis de contenido como método de investigación», en XXI. Revista de educación, 2002, n.4, 167-180.

Marzal, M.A. y Moreira, J.A., (2001): «Modelos teóricos y elementos funcionales para el análisis de contenido documental: definición y tendencias», en Investigación bibliotecológica, n.15, (31), 125-162.

Neuendorf, K.A., (2002): The Content Analisis Guidebook, Thousand Oaks, London, New Delhi: Sage.

Piñuel, J.L., (2002): «Epistemología, metodología y técnicas del análisis de contenido», en Estudios de Sociolingüística 3(1), pp. 1-42, http://personales.jet.es/pinuel.raigada/A.Contenido.pdf

Poibeau, T. \& Dutoit, D., (2002): «Inferring knowledge from a large semantic network», Proceeding of the Semantic networks workshop, during the Computational Linguistics Conference (COLING 2002), Taipei, Taiwan.

West, M. D. (ed.)., (2001): Theory, Method, and Practice in Computer Content Analysis. (Progress in Communication Sciences, Volume 16). Westport, CT/London: Ablex Publishing.

\section{Anexo: Diccionario (campos conceptuales para el análisis automático)}

Argentina $=$ Argentina, argentinos

audiovisual $=$ audiovisual

Brasil = Brasil, Brazil

Canarias $=$ Canarias, canario, canarios

ciber = ciber, cibercultura, cibermedios, cibernética, ciberperiodismo, ciberusuarios ciencia = ciencia, ciencias, científica, científico, científicos

cine = cine, cinefilia, cinematografía, cinematográfica, cinematográficas, cinematográfico,

fílmico, fílmografía

comunicación = comunicación, comunicacional, comunicaciones, comunicar, comunicativa,

comunicativo, comunicativos

comunicador $=$ comunicador, comunicadora, comunicadores, comunicólogos, informadores

Costa-Rica $=$ Costa-Rica, costarricense, costarricenses 
Cuba $=$ Cuba

cultura $=$ cultura, cultural, culturales, culturas

democracia = democracia, demócratas, democrática, democrático, democratización

desarrollo = desarrollo

digital = digital, digitales, digitalización

discurso = discursiva, discursivas, discurso

economía = economía, económica, económico

edición $=$ edición, ediciones

educación = docencia, educación, educar, educativa, educativo, enseñanza, escolar,

escolares, estudiantes, formación, profesor, profesorado

El-País = El-País

elección = elección, elecciones, electoral, electorales

España = España, español, española, españoles

Estados-Unidos = EEUU, Estados-Unidos, USA

ética = deontología, deontológicos, ética, Ética, éticos, valor, valorativa, valores

fotografía = fotografía, fotográfica, fotógrafos, foto, fotos

global = global, globalidad, globalización

gráfica = dibujo, gráfica, gráfico, gráficos, grafismo, grafistas

guerra = guerra

hipermedia $=$ hipermedia, hipermedial, hipermedio, hipertexto, hipertextual

historia = historia, histórica, históricas, histórico, históricos

iconografía = iconicidad, icónico, icónico-verbal, icónicos, iconografía, iconográfico,

ideografía, imagen, imágenes

identidad $=$ identidad, identidades, identificación

industria = industria, industrial, industrializados

infografía = infografía, infográficos, infoperiodismo, megagráficos, megainfografía

información = información, informativa, informativas, informativo, informativos

informática = computador, informática, ordenador

internet = en-línea, enlínea, internet, Internet2, on-line, red

investigación = investigación

local $=$ local

medio = media, medias, mediática, mediático, mediáticos, medio, medio-portal, medios

mensaje $=$ mensaje, mensajes

mercado $=$ mercado, mercados, comercio, comercial

México = mexicana, mexicanos, México, México

noticia $=$ noticia, noticias, noticieros, noticioso

nuevo = nueva, nuevas, nuevo, nuevos

periodismo $=$ fotoperiodismo, periodismo, periodista, periodistas

política $=$ política, políticas, político, políticos

prensa = diario, diarios, imprenta, impreso, impresos, periódicos, periodística, periodístico,

prensa, publicaciones, revista, revistas, semanario

propaganda $=$ propaganda, propagandistas, propagandístico 
publicidad $=$ publicidad, publicitaria, publicitario público = pública, públicas, público, públicos radio = radio, radiofónic, radiofónicas, radiofónico red-social $=$ red-social

social = social, sociales tecnología = tecnología, tecnologías, tecnológica, tecnológico, tecnológicos televisión = televisión, televisivas, televisivo, televisivos, televisoras, televisual, TV teoría = teoría, teorías, teórica

Venezuela = venezolana, venezolano, venezolanos, Venezuela web $=$ web, http 2-0 = 2-0 [Se usa el guión en lugar del punto dado que éste siempre separa oraciones]

\section{Forma de citar este artículo en bibliografías}

COLLE, Raymond (2012): "La temática de las ponencias del III Congreso Latina de Comunicación Social”, en Revista PANGEA, 3, páginas 1 a 22. Red Académica Iberoamericana de Comunicación. Recuperado el __ de de 2 de: http://revistapangea.org 